de leur maturité sexuelle retardée, ont besoin d'une quantité d'aliments plus faible pour atteindre l'âge de la ponte. Les études de nutrition ont porté sur l'influence :

1. des taux de calcium,

2. du doublement du taux normal de vitamines et d'oligo-éléments,

3. du taux protéïque jusqu'à 21 p. 100 ,

4. de la supplémentation en méthionine, lysine et acide linoléïque.

Quoique la production d'œufs ait plafonné à partir du taux protéique de 15 p. 100, la taille des œufs a continué à augmenter jusqu'à 21 p. 100 . L'addition de 0,05 p. 100 de méthionine a augmenté la ponte et le poids des œufs significativement et à des niveaux comparables à ceux observés avec une ration à 21 p. 100 de protéines. Actuellement, un faible taux de protamone est donné aux pondeuses élevées avec ou sans supplément de protamone, mais ce taux a dû être réduit de moitié, jusqu'à 0,0167 p. 100 , car il abaissait la ponte.

Les études physiologiques ont inclus l'observation des hypophyses, thyroïdes, surrénales et ovaires, à la fois pour des femelles en croissance et adultes, ainsi qu'un test biologique d'homogénats hypophysaires sur des rates immatures hypophysectomisées. Quoique les thyroïdes des femelles naines soient apparues plus petites que celles des normales à 7-13 mois d'âge, le test biologique n'a pu prouver que les modifications thyroïdiennes chez les naines soient dues à des altérations de l'activité thyréotrope de leur hypophyse.

Le résultat du test biologique des hypophyses ne confirme pas l'hypothèse de l'accumulation d'hormone somatotrope dans les hypophyses d'oiseaux nains. L'observation de l'ACTH et de l'activité gonatrodope a été faite aussi sur les hypophyses naines.

\title{
EFFET DE GÈNES LIÉS AU SEXE SUR LA TAILLE CORPORELIL ETT LA REPRODUCTION
}

\author{
R. GEORGE JAAP \\ Ohio State University \\ Colombus. Ohio 432 Io (U.S.A.)
}

Deux gènes au moins, situés sur le chromosome sexuel, réduisent la vitesse de croissance et la taille arlulte des poules. L'un est le gène de nanisme lié au sexe bien connu $d w$.Nous employons le symbole $B$ pour un autre gène lié au sexe parce qu'on le trouve habituellement dans les races Bantam. Les données présentes indiquent que $B$ et $d w$ peuvent être allèles, l'ordre de dominance décroissant chez les mâles hétérozygotes étant + (normal), $B$ et $d w$. Quand le poids corporel des femelles normales $(+)$ est d'environ $2 \mathrm{~kg}$, les femelles $d w$ atteignent 65 à 70 p. 10 de ce poids et les femelles $B 90$ p. 100 au moins du poids adulte des femelles normales. Dans les populations de type broiler où le poids adulte des femelles normales approche $4 \mathrm{~kg}$ ou plus, les femelles $d w$ atteignent 80 p. 100 de ce poids normal, contre $65-70$ p. 100 chez le type "ponte " de plus petite taille.

Les femelles naines $d w$ sont habituellement identifiables non seulement par leur petite taille, mais aussi par leurs tarses courts. Par contraste, les femelles $B$ ne peuvent être phénotypiquement distinguées des femelles normales et sont identifiables seulement lorsque le chromosome sexuel portant $B$ contient un marqueur phénotypique visible tel que l'emplumement rapide ou lent, ou la couleur du duvet argentée ou dorée. Le locus $I d$ (inhibiteur de la mélanine dermique), qui est presque indépendant des loci $S K$, ne permet pas d'identifier l'effet de $B$. 
Chez les femelles de type "ponte", $d w$ réduit l'intensité de ponte beaucoup plus que $B$; cependant, cette réduction plus grande de la production d'œufs par $d w$ pourrait être attribuée à une plus petite taille corporelle per se, de façon semblable à la réduction de ponte chez les femelles dont la petite taille est due à l'effet de gènes autosomaux.

La production d'œufs à couver par poule de type "broiler " est faible, en partie à cause d'un syndrôme de surplus " de jaune ". L'allèle $d w$ remédie à ce syndrôme et diminue le nombre d'œufs défectueux, sans réduire le nombre total d'ovipositions comme c'est le cas chez les femelles de type "ponte".

L'allèle $d w$ réduit légèrement moins la taille des œufs chez les mères de type "broiler " que chez les femelles de type "pondeuse ". Une réduction du poids de l'œuf de quatre grammes attribuable à $d w$ chez les mères "broiler " diminue le poids à 8 et à 12 semaines de leur descendance naine. Par contraste, lorsque ces mères $d w$ type "broiler "sont accouplées à des pères du même type et de grande taille $(+)$, leur descendance femelles atteint des poids à 8 semaines é gaux ou supérieurs à ceux de mères normales accouplées aux mêmes pères. Quoique le poids corporel à 8 semaines des mâles hétérozygotes $(+d w)$ issus de mères naines soit inférieur à celui des mâles homozygotes $(++)$ de mères normale, la réduction correspondante observée est faible et pourrait ne pas être statistiquement significative.

La fertilité et le taux d'éclosion des œufs pondus par des femelles naines inséminées artificiellement est apparue égale ou supérieure à celle des normales. La production de viande de poulet de chair par mère naine doit être égale ou légèrement supérieure à celle des mères normales possédant l'allèle + au locus $d w$. Le coût de croissance et d'entretien de la mère naine de type "chair " a été estimé à environ " un " $\left.{ }^{1}\right)$ cent de moins par poulet, ce qui équivaut à abaisser de plus de 2. 500 \$ le cout de production de poulets par homme et par an.

Nous explorons la possibilité d'interactions épistatiques favorables ou défavorables entre $d w$ et le type de génômes désirables pour une production économique des oeufs et des poulets. Une autre question consiste à savoir si $d w$ ou $B$ peut aider à révéler une variabilité génétique cachée utilisable pour l'amélioration génétique des populations de type "ponte " ou "chair ".

\author{
QUELQUES EFFETS DU GÈNE $d w$ SUR LA PONTE \\ ET SUR IA QUALITÉ, DES GUFS \\ Laboratoive de Génétique factorielle, \\ Centre national de Recherches zootechniques, I.N.R.A., \\ 78 - Jouy-en-Josas (France)
}

Dans une souche expérimentale légère en ségrégation au locus $D w$, l'allèle $d w$ s'accompagne d'un âge au $1^{\text {er }}$ œuf légèrement retardé. Ceci n'est sans doute pas explicable par un effet de "compétition " sociale. Parallèlement, la croissance du testicule est plus lente chez les coquelets "nains " que chez leurs frères "normaux ".

L'intensité de ponte est réduite en présence de $d w$ (de l'ordre de 15 p. 100 dans notre population). La longueur moyenne des séries de ponte est abaissée, et la diminution du poids de l'œuf du début à la fin d'une série est plus importante chez les poules naines que chez leurs sœurs normales. Ceci pourrait refléter une vitellogenèse moins active, en accord avec les observations de JAAP (1968).

Outre leur poids, diverses caractéristiques des œufs sont modifiées par le gène dw : la pigmentation des coquilles est moins intense, ainsi que celle du jaune ; l'albumen est plus consistant en moyenne. Les effets de ce gène semblent ainsi s'exercer à des stades variés de la formation de l'œuf. Par contre, il ne modifie pas la proportion relative du jaune et du blanc.

(1) Un " cent " $=5$ à 6 centimes français. 